\title{
Can we probe the Lorentz factor of gamma-ray bursts from GeV-TeV spectra integrated over internal shocks?
}

\author{
Junichi Aoi ${ }^{1}$, Kohta Murase ${ }^{2,3}$, Keitaro Takahashi ${ }^{4}$, Kunihito Ioka ${ }^{5}$ and Shigehiro Nagataki ${ }^{1}$
}

\begin{abstract}
We revisit the high-energy spectral cutoff originating from the electron-positron pair creation in the prompt phase of gamma-ray bursts (GRBs) with numerical and analytical calculations. We show that the conventional exponential and/or broken power law cutoff should be drastically modified to a shallower broken power-law in practical observations that integrate emissions from different internal shocks. Since the steepening is tiny for observations, this "smearing" effect can generally reduce the previous estimates of the Lorentz factor of the GRB outflows. We apply our formulation to GRB 080916C, recently detected by the Large Area Telescope detector on the Fermi satellite, and find that the minimum Lorentz factor can be $\sim 600$ (or even smaller values), which is below but consistent with the previous result of $\sim 900$. Observing the steepening energy (so-called "pair-break energy") is crucial to diagnose the Lorentz factor and/or the emission site in the future observations, especially current and future Cherenkov telescopes such as MAGIC, VERITAS, and CTA.
\end{abstract}

Subject headings: acceleration of particles - gamma-ray burst: general - opacity - radiation mechanisms: non-thermal - shock waves

\section{Introduction}

Gamma-ray burst (GRB) is one of the most mysterious objects in the universe. The typical energy of its prompt emission is $\sim 100-1000$ $\mathrm{keV}$, which corresponds to the breaking point of the broken power law describing the energy spectrum well (Band et al. 1993). Although there have been many works on the mechanism of prompt emission since its discovery, it is still unknown. Combined with the optically thin synchrotron emission mechanism, the internal shock model has been most widely discussed to explain the prompt emission (see, e.g., Mészáros 2006; Zhang

\footnotetext{
${ }^{1}$ YITP, Kyoto University, Kyoto, 606-8502, Japan

${ }^{2}$ Department of Physics, Tokyo Institute of Technology, 2-12-1, Ookayama, Meguro-ku, Tokyo, 152-8550, Japan

${ }^{3}$ Center for Cosmology and AstroParticle Physics, 191 West Woodruff Avenue, Columbus, OH 43210, USA; murase.2@mps.ohio-state.edu

${ }^{4}$ Department of Physics and Astrophysics, Nagoya University, Nagoya, 464-8602, Japan

${ }^{5}$ KEK (High Energy Accelerator Research Organization), Tsukuba, 305-0801, Japan
}

2007; Fan \& Piran 2008, for reviews). In fact, this model can reproduce the behavior of complicated light curves (e.g., Kobavashi et al. 1997; Daigne \& Mochkovitch 1998; Mimica et al. 2007; Bošnjak et al. 2009). The complicated light curve consists of several pulses and these pulses include sub-pulses which vary violently. Collisions among inhomogeneous outflows lead to the shock formation that converts the kinetic energy of the outflow to the internal energy. We can interpret the non-thermal gamma-ray emission as the emission of electrons which are accelerated at the shocks, where inhomogeneous outflows characterized by multiple sub-shells can easily produce highly variable light curves.

High-energy emission from GRBs was already detected by EGRET before Fermi, but it has not been clear where the end point of the high-energy tail is (e.g., Schneid et al. 1992; Sommer et al. 1994; Hurley et al. 1994; Schneid et al. 1995). If the spectrum extends to higher-energy range, sufficiently high-energy photons cannot avoid the electron-positron pair- creation process. Thus, 
it has been expected that there should be a cutoff due to this process, although there was no crucial observational evidence on it. The paircreation cutoff energy generally depends on the emission radius $r$ and the bulk Lorentz factor of the outflow $\Gamma$. Hence, we can obtain information on these quantities once it is observed. Therefore, many authors investigated the possibility to extract the information from the cutoff energy (e.g. Baring \& Harding 1997; Lithwick \& Sari 2001; Razzaque et al. 2004; Murase \& Ioka 2008; Gupta \& Zhang 2008; Granot et al. 2008).

However, these studies focused on the highenergy emission from one emission zone, neglecting the time evolution of physical quantities during the emission. In realistic observations, it is likely that the observed emission comes from many emission regions, and physical quantities should be time dependent even during one sub-pulse produced by one emission zone. Hence, the cutoff behavior in spectra should be affected by both (1) the time-evolution effect during one sub-pulse and (2) the time-integration effect over many subpulses. On the former point (1), the time variation of properties on the photon field during the sub-shell-crossing timescale $(\delta t /(1+z) \sim l / c)$ is significant and comparable to that during the total duration of the emission (Granot et al. 2008; Bošnjak et al. 2009), which can alter the resulting high-energy spectrum. The cutoff also evolves reflecting the time evolution of the emission site and the Lorentz factor of a sub-shell (Baring 2006). The latter point (2) is also important, since the observed high-energy spectrum should be different from that expected in the one-zone treatment unless we can separate the contribution of each emission zone. More specifically, in the internal shock model, we would see the superposition of the emission from many collisions among different sub-shells during the total duration. Especially, if a flux of a single sub-pulse is small, we cannot avoid integrating the contribution of all the subpulses to observe the burst. In such a case, it is important to study the effect of a lot of internal collisions.

In this paper, we focus on the above timeintegration effect over many sub-pulses in the internal shock model, and examine whether we can extract information about the bulk Lorentz factor of GRBs from high-energy GeV-TeV spectra. We model the high-energy emission from the multiple emission regions caused by multiple collisions, and calculate energy spectra numerically. We need to consider a lot of sub-shells with various Lorentz factors to explain complicated and irregular light curves. Our numerical approach can treat this situation well that is valid even when the variance of the distribution of sub-shells' Lorentz factors is large. This is an advantage over the analytical approach which is valid only when the variance is small enough (see, e.g., Li 2010). In fact, the variance should be large in the internal shock model, in order that the sub-shells' kinetic energy is efficiently converted into the radiation energy as prompt emission Kobavashi et al. 1997; Kobayashi \& Sari 2001). For calculations, we simplify the emission from each collision using a rather phenomenological procedure. This may be too simple but enough to study the smearing effect by many internal collisions. Other observational effects coming from radiation mechanisms will be discussed later.

In Section 2, we explain the calculational method used in this work. We consider multiple sub-shells and integrate the energy spectrum of emission which comes from each merged shell. In Section 3, we show the results on the energy spectrum of GRBs. In Section 4, we discuss the possibility to extract information about the Lorentz factor of the GRB outflow from observations. Moreover, we discuss the case of GRB080916C, which is an interesting event to study (Zou et al. 2009; Wang et al. 2009; Zhang \& Pe'er 2009). We also compare our numerical results with the analytical results. In Appendices A and B, we explain the dynamics of shells which approximate the inhomogeneous outflows of GRBs.

\section{Method}

The inhomogeneous outflow is characterized by multiple sub-shells. For simplicity, we only consider completely inelastic collisions between shells and neglect the interaction via pressure waves (which is a good approximation if the outflow energy is dominated by the kinetic part as shown in (Daigne \& Mochkovitch 2000)). The kinetic energy of the shells is converted to the radiation energy via electron acceleration in the internal shock model, where we may phenomenologically 
give the energy spectrum of emission from each merged shell (see Section 2.1). In Appendices $\mathrm{A}$ and $\mathrm{B}$, we summarize the dynamics of multiple collisions which was similar to that given in Kobavashi et al. (1997). As described in Section 2.2, we can evaluate the cutoff energy of the spectrum, which originates from the electron-positron pair-creation process. Hereafter, we express physical quantities that are defined in the comoving frame of the outflow with primes (e.g., photon energy $\left.\varepsilon^{\prime}\right)$ and those which are defined in the source (or laboratory) frame without primes (e.g., $\varepsilon$ ).

\subsection{Radiation from a Merged Shell}

The internal energy $E_{\text {int }}$ is determined when two shells merge (see the Appendix A). This energy is divided into the energy of protons, electrons and magnetic fields. The parameter $\epsilon_{p}$ indicates the fraction of the energy in (thermal and nonthermal) protons. Similarly, the energies of (nonthermal) electrons and magnetic fields are characterized by $\epsilon_{e}$ and $\epsilon_{B}$, respectively. For simplicity, we just assume $\epsilon_{e}=\epsilon_{B}=\epsilon_{p}=1 / 3$. In addition, we consider the fast cooling case, where almost all the energy of electrons accelerated in each merged shell is released as radiation (e.g., Guetta \& Granot 2003).

Observed energy spectra of prompt emission are usually fitted well by broken power-law ones (Band et al. 1993). This broken power-law spectrum is characterized by low-energy power-law index $\alpha$, high-energy power-law index $\beta$, and the break energy $\varepsilon_{b}$. Therefore, we assume that the energy spectrum for each collision is expressed by the broken power law, as expected for the synchrotron and/or inverse-Compton emission from nonthermal electrons. Although there may be an additional component in the high-energy spectrum (e.g., González et al. 2003, see also later discussions in Section 4.4), we approximate the spectrum using the broken power-law spectrum for the demonstrative purpose. We here adopt $\alpha=1, \beta=$ 2.2 , and $\varepsilon_{b}=300 \mathrm{keV}$, which are typical values obtained from observations (e.g., Preece et al. 2000). Given the spectral shape, we can normalize a photon distribution function $d n / d \varepsilon$ by the total radiation energy $\epsilon_{e} E_{\text {int }}$ with

$$
\epsilon_{e} E_{\mathrm{int}}=(1+z) V \int d \varepsilon \varepsilon \frac{d n}{d \varepsilon},
$$

where $V$ is the volume of each emission region (see the Appendix A). The distribution function of photons in the source frame is given by

$$
\frac{d n}{d \varepsilon}= \begin{cases}K\left(\frac{\varepsilon}{\varepsilon_{b}}\right)^{-\alpha} & \left(\varepsilon<\varepsilon_{b}\right) \\ K\left(\frac{\varepsilon}{\varepsilon_{b}}\right)^{-\beta} & \left(\varepsilon \geq \varepsilon_{b}\right) .\end{cases}
$$

Here, $K$ is the normalization factor which is determined by Equation (1). For our calculations, we use the minimum energy of $300 \mathrm{eV}$ (which is likely to come from the self-absorption process) and the maximum energy of $10^{13} \mathrm{eV}$ (which roughly corresponds to the most optimistic energy of accelerated electrons) before considering photon attenuation by pair creation. Note that the results in our study are insensitive to these values as long as $\alpha<2$ and $\beta>2$.

\subsection{The Pair-creation Cutoff}

High-energy photons cannot escape from a merged shell because of the pair-creation processes such as $\gamma \gamma \rightarrow e^{+} e^{-}$and $e \gamma \rightarrow e e^{+} e^{-}$. The most important process is $\gamma \gamma \rightarrow e^{+} e^{-}$under typical conditions of GRBs (Razzaque et al. 2004). We calculate the pair-creation cutoff energy for highenergy photons by considering only $\gamma \gamma \rightarrow e^{+} e^{-}$.

The optical depth $\tau_{\gamma \gamma}$ for this process at some energy $\varepsilon^{\prime}$ can be calculated for a given photon spectrum. We assume the power-law spectrum in Section 2.1 and calculate the optical depth in the comoving frame of the outflow as follows (Gould \& Schréder 1967; Lightman \& Zdziarski 1987; Svensson 1987; Lithwick \& Sari 2001; Baring 2006; Murase \& Ioka 2008):

$$
\begin{array}{r}
\tau_{\gamma \gamma}\left(\varepsilon^{\prime}\right) \simeq \xi(\beta) n^{\prime}\left(\tilde{\varepsilon}^{\prime}\right) \sigma_{T} \Delta^{\prime}, \\
{\left[\tilde{\varepsilon}^{\prime}=\frac{\left(m_{e} c^{2}\right)^{2}}{\varepsilon^{\prime}}\right],}
\end{array}
$$

where the number density of photons whose energies are larger than $\tilde{\varepsilon}^{\prime}$ is given by

$$
n^{\prime}\left(\tilde{\varepsilon}^{\prime}\right) \simeq K \int_{\tilde{\varepsilon}^{\prime}}\left(\frac{\varepsilon^{\prime}}{\varepsilon_{b}^{\prime}}\right)^{-\beta} d \varepsilon^{\prime} .
$$

Here $\tilde{\varepsilon}^{\prime}$ is the energy of a photon which interacts with the photon of energy $\varepsilon^{\prime}$ at the pair-creation threshold, and $\sigma_{T}$ is the Thomson cross section. Note that Equation (4) is valid when $\tilde{\varepsilon}^{\prime}$ satisfies 
$\tilde{\varepsilon}^{\prime}>\varepsilon_{b}^{\prime}$, and we may approximate the optical depth as $\tau_{\gamma \gamma}\left(\varepsilon^{\prime}\right) \simeq \tau_{\gamma \gamma}\left(\tilde{\varepsilon}_{b}^{\prime}\right)$ for $\tilde{\varepsilon}^{\prime}<\varepsilon_{b}^{\prime}$ if the spectrum is typical $\alpha \sim 1$ (see for more discussions in Sections 3 and (4). The number density of photons in the comoving frame $n^{\prime}$ relates with the number density in the source frame $n$ as $n^{\prime}\left(\varepsilon^{\prime}\right) \simeq n\left(\varepsilon^{\prime} \Gamma\right) / \Gamma$ because the isotropically distributed photons, whose energy is $\varepsilon^{\prime}$ in the comoving frame, are blueshifted to $\varepsilon \simeq \Gamma \varepsilon^{\prime}$ on average. $\xi(\beta)$ is the numerical factor that depends on the power-law index and this factor decreases with $\beta$; the values are $\xi(\beta)=11 / 90 \simeq 0.12$ and $\xi(\beta)=7 / 75 \simeq 0.093$ for $\beta=2$ and $\beta=3$, respectively Svensson 1987). We can use $\xi(\beta) \simeq 7(\beta-1) /\left[6 \beta^{5 / 3}(\beta+1)\right]$ for $1<\beta<7$ for isotropically distributed photons (Baring 2006). We approximate the width of the emission region $\Delta^{\prime}$ by using the width of the merged shell, that is, $\Delta^{\prime} \simeq l_{m}^{\prime}$ (see the Appendix A). The pair-creation cutoff energy $\varepsilon_{\text {cut }}^{\prime}$ is defined as the energy at which the optical depth becomes unity, $\tau_{\gamma \gamma}\left(\varepsilon_{\text {cut }}^{\prime}\right)=1$.

For sufficiently small emission radii or Lorentz factors, the Thomson optical depth $\tau_{T}$ may exceed unity. Even in such cases, we always expect that the pair-creation cutoff should be comparable to $\sim \Gamma m_{e} c^{2} /(1+z)$. However, the Compton scattering is very important when $\tau_{T} \geq 1$ (see, e.g., Thompson 1994; Pe'er \& Waxman 2004; Rees \& Mészáros 2005). The photon spectrum may have a thermal or quasi-thermal component (see, e.g., Pe'er \& Waxman 2004; Pe'er et al. 2006). It may deviate from the power-law shape and even have the pair annihilation bump (Ioka et al. 2007; Murase \& Ioka 2008). In this work, we treat the cutoff as $\varepsilon_{\text {cut }}=\varepsilon_{\text {ann }}=$ $\Gamma m_{e} c^{2} /(1+z)$ for $\tau_{\gamma \gamma}\left(\Gamma m_{e} c^{2} /(1+z)\right)>1$ for simplicity. Although this may not be a good approximation, we expect that this treatment is enough for our purpose since we do not have so many shell collisions with $\tau_{\gamma \gamma}\left(\Gamma m_{e} c^{2} /(1+z)\right)>1$ for our adopted parameter sets.

For sufficiently large emission radii or Lorentz factors, both the Thomson optical depth and the pair-creation optical depth may be very small, where no pair-creation cutoff exists because of $\tau_{\gamma \gamma}\left(\varepsilon^{\prime}\right)<1$ for any energy $\varepsilon^{\prime}$. Then, a significant fraction of $\gtrsim \mathrm{TeV}$ photons escapes from the source without internal attenuation. These photons should be attenuated via pair creation by the cosmic microwave background (CMB) and cos- mic infrared background (CIB) photons (see, e.g., Murase et al. 2007, and references therein). We include this effect by using the "low-IR" model of Kneiske et al. (2004). Note that recent observations of $\mathrm{TeV}$ blazars imply that the low-IR model is favored (Aharonian et al. 2006, 2007). The created pairs can affect the primary spectrum itself via the inverse Compton scattering of the CMB photons (Dai \& Lu 2002; Razzaque et al. 2004; Murase et al. 2007). However, this effect leads to relatively minor effects during the prompt emission and can be important in the late time only when the intergalactic magnetic field is weak enough (Murase et al. 2008; Takahashi et al. 2008; Murase et al. 2009). Hence, we neglect this possible sub-dominant contribution from the pair echo emission in this work.

\subsection{Parameters for Shells}

We have the following parameters in our calculations: the initial length between two shells $d$, the initial width of a shell $l$, the number of shells $N$, the distance from GRB to the Earth $D$ (or the redshift $z$ ), the fraction of energy that goes to non-thermal electrons $\epsilon_{e}$, the initial number density of protons in the innermost shells $n_{p}$, and the most inner radius of sub-shells $r_{\text {ini }}$ (see also the Appendix B). We adjust the number density $n_{p}$ so as to have the given isotropic luminosity $L_{\gamma}$. In addition, we assume the log-normal distribution of shell's bulk Lorentz factors $\Gamma$, whose average value is $\Gamma_{0}$, with the fluctuation $A$. Note that $\Gamma_{0}$ is different from the mathematically strict mean value (see the Appendix B for definition of the log-normal distribution). We choose the lognormal distribution for a demonstrative purpose, although there is no observational evidence to support this distribution. However, one can see that the result does not change so much even if we use some other distributions (e.g., homogeneous distributions). Note that our aim here is not to reproduce observational relations such as a correlation between the peak energy in a spectrum and the isotropic luminosity in a burst, called the $\varepsilon_{b}-L_{\gamma}$ relation (Liang et al. 2004; Yonetoku et al. 2004). Examining distributions of parameters, taking into account such observational relations, would be important because it may include the information of the Lorentz factor distribution. But, such studies are beyond the scope of this work 


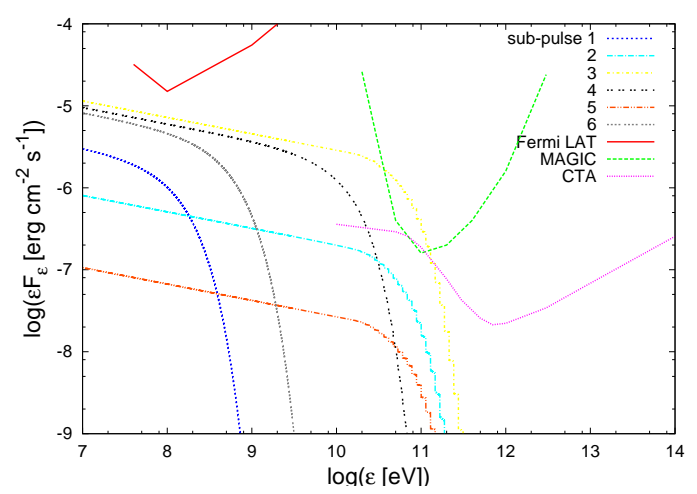

Fig. 1.- $-\varepsilon F_{\varepsilon}$ spectrum of prompt emission from each two-shell collision where six spectra are randomly selected from dozens. The number of shells is set to $N=100$ and the distribution of initial Lorentz factors is log-normal with $\Gamma_{0}=500$ and an initial fluctuation $A=1$, which are defined by Equation (B1). Other parameters are the initial distance between shells $d=5 \times 10^{9} \mathrm{~cm}$, the initial thickness of a shell $l=10^{9} \mathrm{~cm}$, the initial radius of the innermost shell $r_{\mathrm{ini}}=10^{11} \mathrm{~cm}$, the energy fraction of electrons $\epsilon_{e}=1 / 3$, and the redshift $z=1$. We adjust the initial number density $n_{p}$ so as to have the mean luminosity of $L_{\gamma}=10^{52}$ $\mathrm{erg} \mathrm{s}^{-1}$. The sensitivity curves of LAT on Fermi, MAGIC, and CTA are also shown.

so that we just assume the log-normal distribution. In this paper, the $\Lambda$ CDM cosmology with $\Omega_{m}=0.3, \Omega_{\Lambda}=0.7$, and $H_{0}=70 \mathrm{~km} \mathrm{~s}^{-1} \mathrm{Mpc}^{-1}$ is also adopted.

\section{Results}

In Figure1, we show the energy spectra of emission from each two-shell collision using the method in Section 2. We only show six spectra randomly selected from a GRB to make the figure easy to see, although there are dozens of spectra for one realization. We can see the electron-positron pair-creation cutoff in each spectrum. One may expect to obtain much information about the GRB if we can observe the cutoff energy. However, we have to integrate these spectra unless we can observe these spectra separately due to the lack of photon numbers. In Figure 1 all the spectra are below the sensitivity curve of Fermi ( the cutoff energy may be observed by MAGIC and/or CTA. But note that these observatories take a few

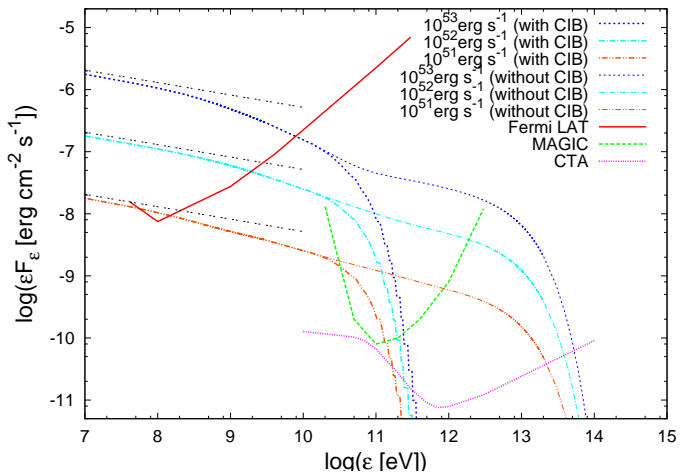

Fig. 2.- Time-integrated spectra for different luminosities. We calculate three cases with almost the same pair-break energy (defined in Section 3) but different luminosities $L_{\gamma}=10^{53}, 10^{52}$, and $10^{51} \mathrm{erg} \mathrm{s}^{-1}$. The fluctuation of the initial Lorentz factor $A$ is 0.5 for $L_{\gamma}=10^{53} \mathrm{erg} \mathrm{s}^{-1}, 0.7$ for $10^{52} \mathrm{erg} \mathrm{s}^{-1}$, and 1.6 for $10^{51} \mathrm{erg} \mathrm{s}^{-1}$, respectively. Other parameters are the same as Figure 1 except for the number density $n_{p}$. The number density is determined to set the luminosity to the above values. The slope of $\varepsilon^{-0.2}$ is shown with the dotted line for comparison. The sensitivity curves of LAT on Fermi, MAGIC, and CTA are also shown.

dozens of seconds to turn the detector to the direction of a GRB after an alert from a space telescope). In this case, only the integrated spectrum is observable and the cutoff is smeared out as we explain below.

Here, the sensitivity curve of Fermi is calculated from the effective area 1 under the criterion that at least five photons are collected. The sensitivity curve of MAGIC is roughly estimated from the effective area for a zenith angle of $20^{\circ}$ with the criterion that at least 10 photons are collected, although the actual detectability requires careful analysis (Albert et al. 2007). The sensitivity of CTA is also estimated from the public curve (Wagner et al. 2009). Although the actual one depends on details that are not available, it is shown just for demonstration by raising the public one by $\sqrt{50 \mathrm{hr} / 20(1+z) \mathrm{s}}$.

In Figure 2, the three time-integrated spectra are shown whose isotropic luminosities are $10^{53}$

${ }^{1}$ Shown at http://www-glast.slac.stanford.edu/software/IS/glast_ lat _ performance.htm. 
(a)

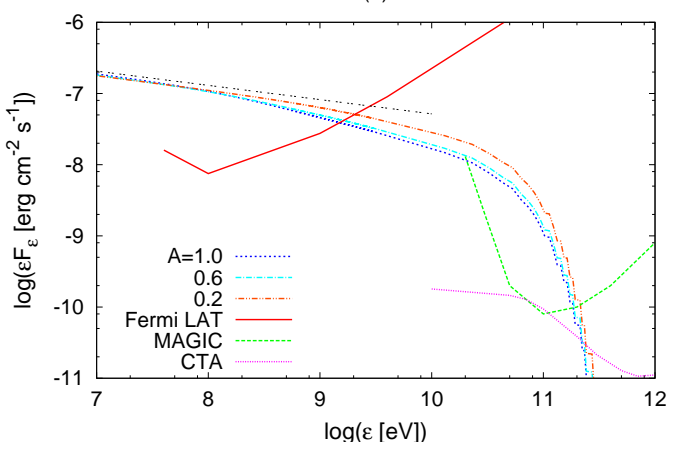

(c)

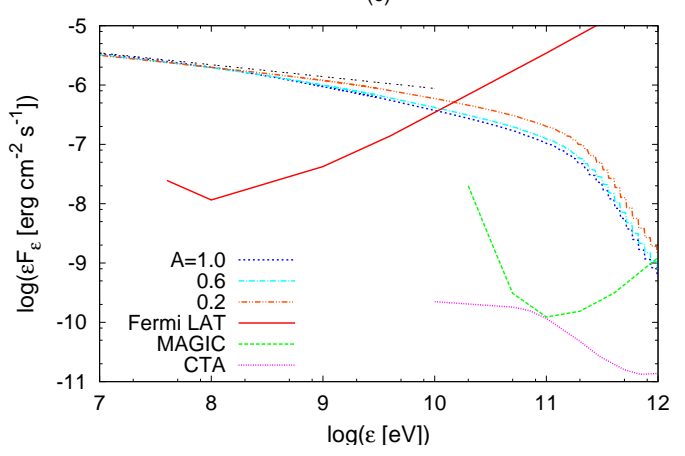

(b)

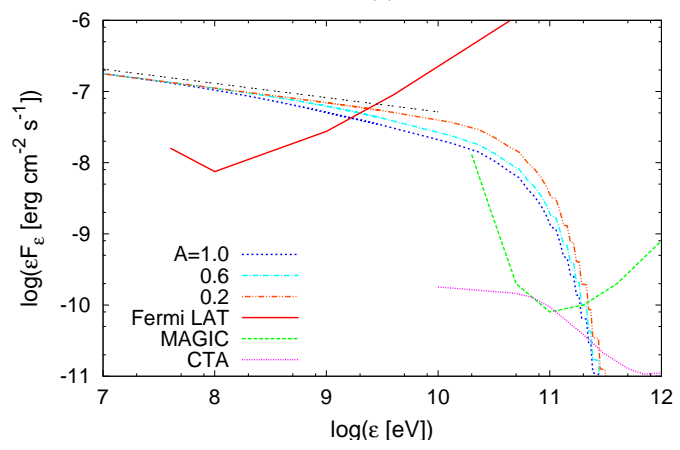

(d)

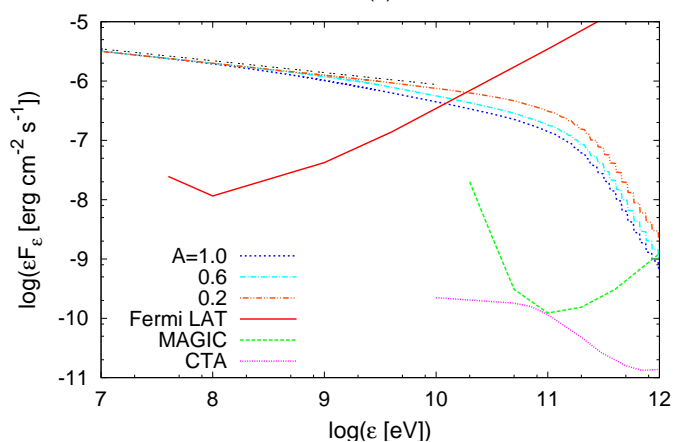

Fig. 3. - Time-integrated spectra for the different fluctuation of Lorentz factor $A=1,0.6,0.2$. The luminosity is set to $L_{\gamma}=10^{52} \mathrm{erg} \mathrm{s}^{-1}$ in these figures. Other parameters are as follows: (a) $z=1, l=10^{8} \mathrm{~cm}$, $N=10^{3}$; (b) $z=1, l=10^{9} \mathrm{~cm}, N=10^{2}$; (c) $z=0.3, l=10^{8} \mathrm{~cm}, N=10^{3}$; (d) $z=0.3, l=10^{9} \mathrm{~cm}$, $N=10^{2}$. We have $\Gamma_{0}=500, d=5 \times l, r_{\text {ini }}=10^{11} \mathrm{~cm}$, and $\epsilon_{\mathrm{e}}=1 / 3$ for all cases. The dotted line shows the slope of $\varepsilon^{-0.2}$ for comparison. We consider the effect of CIB in TeV energy range. The sensitivity curves of LAT on Fermi, MAGIC, and CTA are also shown.

$\operatorname{erg~s}^{-1}, 10^{52} \mathrm{erg} \mathrm{s}^{-1}$, and $10^{51} \mathrm{erg} \mathrm{s}^{-1}$. The fluctuation of the initial Lorentz factor $A$ is 0.5 for the case of $10^{53} \mathrm{erg} \mathrm{s}^{-1}, 0.7$ for $10^{52} \mathrm{erg} \mathrm{s}^{-1}$, and 1.6 for $10^{51} \mathrm{erg} \mathrm{s}^{-1}$, respectively. We choose the values of $A$ so as to set the spectrum steepenings at the same energy (see below). Other parameters are described in the caption of the figure. We plot the spectra with and without the CIB effect.

From Figure 2, we can find the following features.

(1) Most importantly, there is no sharp cutoff that originates from the pair production in Figure 2. Instead, the spectral slope becomes steep above a few hundred $\mathrm{MeV}$. We define the beginning point of the steepening as the pair-break energy. This is because the steepening arises from the superposition of the pair creation cutoff in each collision that is plotted in Figure 1. The exponential cutoffs are smeared by the time integration and result in a steepening of the power-law spectrum. Note that this feature is also observed with other distributions of the initial Lorentz factor. Fermi may observe the pair-break energy, if very bright bursts occur in the future. For identifying the steepening feature, it would also be favorable to observe the spectral slope at very high energies with Cherenkov telescopes such as MAGIC and CTA.

(2) The steepening effect is conspicuous for the small fluctuation of the initial Lorentz factor $A$ (corresponding to the high luminosity case $L_{\gamma}=$ $\left.10^{53} \mathrm{erg} \mathrm{s}^{-1}\right)$ while it is not for large $A$. This is because the released energy decreases more rapidly for smaller $A$ as the collision radius of the two shells becomes larger and accordingly as the cutoff energy of a two-shell collision becomes higher. We discuss this effect analytically in Section 4, The 
steepening effect may be observed by Fermi when the luminosity is larger than $\sim 10^{52} \mathrm{erg} \mathrm{s}^{-1}$.

(3) Although the steepening is small for large $A$ at the pair-break energy, the slope gradually becomes softer at larger energy (see also Figure 3). This is because the fluctuation of Lorentz factor evolves smaller with time as shells collide with each other, asymptotically approaching the small $A$ case (see Section 4 for more discussions).

(4) The slope of the spectrum becomes hard, returning back to the original high-energy powerlaw index $\beta$ around TeV energy. This means that the spectrum is produced by a two-shell collision without the pair creation cutoff, i.e., by low-density shells. We can roughly estimate the hardening energy (or the transparent energy) as $\sim \Gamma^{2}\left(m_{e} c^{2}\right)^{2} /(1+z) \varepsilon_{b}$ (see Equation (3) ) in the following way. We assume the photon density is decreasing monotonously above the break energy $\varepsilon_{b}$. Then, as the photon density becomes low with large collision radius and/or small internal energy, the low energy range of the spectrum below $\varepsilon_{b}$ becomes relevant for the pair creation as target photons. However the photon number is insensitive to the photon energy below $\varepsilon_{b}$ for the typical low-energy power-law index $\alpha=1$, and hence the optical depth to the pair creation becomes almost constant for the photon energy above $\sim \Gamma^{2}\left(m_{e} c^{2}\right)^{2} /(1+z) \varepsilon_{b}$. This is why the slope becomes hard above this energy. Unfortunately, it is difficult to observe the hardening effect by the effect of CIB (see below).

(5) There is a cutoff around $10^{13} \mathrm{eV}$. This is not the pair-creation cutoff as discussed above, but the cutoff due to the maximum energy of electrons that we put for convenience. Note that the maximum energy of electrons itself depends on the details of acceleration mechanisms that are not in focus of this work.

(6) If we include the effect of pair-creation interactions with CIB, we have a cutoff around $\mathrm{TeV}$ energy. This effect masks the hardening effect and the maximum energy cutoff discussed above. All those features can be studied by observations of Cherenkov telescopes.

In Figure 3, we show the time-integrated spectra for different fluctuations of initial Lorentz factor $A$ from 1 to 0.2 with the same luminosity $L_{\gamma}=10^{52} \mathrm{erg} \mathrm{s}^{-1}$. We consider the effect of CIB in TeV energy range. The spectrum becomes steep above a pair-break energy. A pair-break energy is low for large $A$ because the collision radius is small and hence the cutoff energy is small for each collision. The steepening is observable for large $A$ even if a GRB occurs at high $z \sim 1$ (Figure $3(\mathrm{a})$ and (b)). We can observe the steepening for small $A$ if the GRB occurs at low $z$ (Figure 3(c) and (d)). We also compare the cases of $N=10^{3}$ $\left(l=10^{8} \mathrm{~cm}\right)$ with that of $N=10^{2}\left(l=10^{9} \mathrm{~cm}\right)$. The shells collide with each other in the inner radius for $N=10^{3}$ than for $N=10^{2}$ because the separation is small. (The separation between the shells is small when the thickness of the shell is small. This is because we assume the separation and the thickness are comparable.) It leads to the smaller pair-creation cutoff in each collision and the pair-break energy becomes smaller.

In Figure 4, we show a change of the power-law index from $\beta=2.2$ due to the effect of the smearing for the case of Figure 3 (a) (the slope in Figure 3) (c) is the same as in Figure 3(a)). We calculate the smeared power-law index by two ways. One is defined by the power-law index between $100 \mathrm{MeV}$ and $1 \mathrm{GeV}$ with the least-squares fitting. This energy range corresponds to the observable energy band in Figure 3(a). Another is defined by the energy range between $1 \mathrm{GeV}$ and $10 \mathrm{GeV}$, which corresponds to the observable energy range in Figure 3(c). Figure 4 shows that the slope in a fixed energy region is steeper for larger $A$. The reason is that the pair-break energy is lower for larger $A$ (see the previous paragraph) and the slope becomes softer as the fixed energy range is more separated from the pair-break energy (see the feature (3) obtained from Figure2), although the steepening is smaller at the pair-break energy for larger $A$ (see the feature (2) obtained from Figure 2). We have more discussions in Section 4.

We also calculate sub-pulse intervals. It is known that the distribution of sub-pulse intervals can be fitted by a log-normal distribution, although there is a small excess at long intervals (McBreen et al. 1994; Li \& Fenimore 1996; Nakar \& Piran 2002; Ioka \& Nakamura 2002). We calculate sub-pulse intervals $\Delta t$ for various average Lorentz factors $\Gamma_{0}$ and fluctuation amplitudes $A$ (see the Appendix B for the definitions). We plot the result for $\Gamma_{0}=500$ and $A=1$ in Figure [5] as the histogram of the logarithmic 


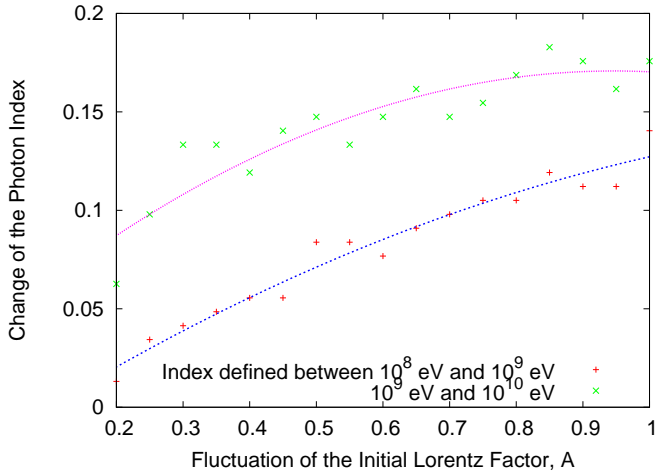

Fig. 4.- Change of the photon index vs. the fluctuation of the initial Lorentz factor $A$. The change of the photon index indicates the difference from the initial high-energy power-law index $\beta$. The photon index is defined in two different energy ranges, $10^{8}-10^{9} \mathrm{eV}$ and $10^{9}-10^{10} \mathrm{eV}$. The spectral index becomes larger as $A$ becomes larger. The solid and dashed lines are quadratic curves obtained from the least-squares fitting.

time intervals. The result is averaged for 100 different random realizations. A sub-pulse interval is defined by an interval between each beginning time of a sub-pulse. We fit the histogram by the log-normal distribution using the method of least squares. A log-normal distribution is characterized by $\mu$ and $\sigma^{2}$ as $f(\Delta t) d \ln \Delta t=$ $\exp \left(-(\ln (\Delta t)-\mu)^{2} / 2 \sigma^{2}\right) / \sqrt{2 \pi \sigma^{2}} d \ln \Delta t$.

In Figure 6, we plot the correlation between the pair-break energy $\varepsilon_{\mathrm{pb}}$ and $\sigma$. We define the pairbreak energy by the energy where the power-law index becomes steeper than $\beta$ by 0.05 . We calculate $\varepsilon_{\mathrm{pb}}$ and $\sigma$ for various $\Gamma_{0}$ and $A$ fixing the luminosity. In this figure, $\varepsilon_{\mathrm{pb}}$ becomes smaller for larger $A$. The cutoff energy of two-shell collision is smaller when the fluctuation is large because the collision radius is smaller and the density of photons is larger. It means $\varepsilon_{\mathrm{pb}}$ becomes small when $A$ is large. It is difficult to observe $\varepsilon_{\mathrm{pb}}$ when the fluctuation is small by the effect of CIB. We discuss the possibility to extract the information from this plot in Section 4 .

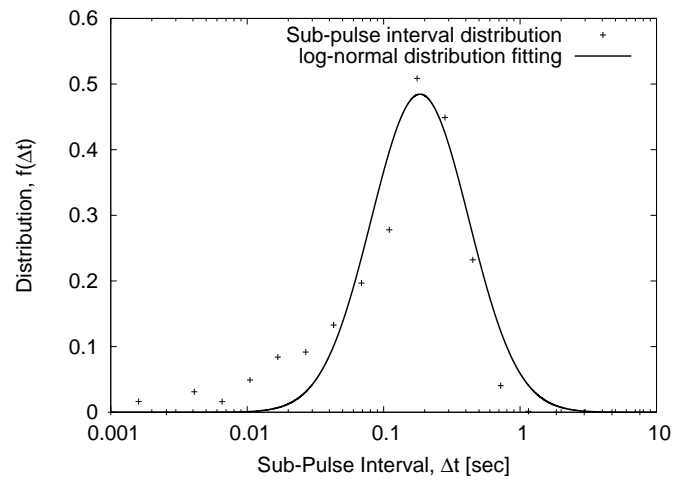

Fig. 5.- Sub-pulse interval distribution. The parameters of the initial Lorentz factor distribution are $\Gamma_{0}=500$ and $A=1$. Other parameters are the same as the calculation in Figure 3(b). We fit the data of sub-pulse intervals by the log-normal distribution with the least-squares method.

\section{Discussion}

\subsection{On Estimate of Lorentz factors}

Even though a pair-creation cutoff energy may give us useful information as we mentioned in Section 1, it does not seem easy to obtain information when the cutoff is masked by the smearing effect (see Figure 7). If our model assumptions are valid, it is difficult to see the obvious cutoff feature in the internal shock model. A clear cutoff feature should be in the very high energies of $\gg \mathrm{GeV}$, but it is likely to be masked due to attenuation by the CIB. Then, can we probe the Lorentz factor from the GRB spectrum? We think that we can still extract useful information on it.

One possibility is that we may constrain the Lorentz factor from the absence of the paircreation cutoff. Since each cutoff is produced by the target photons in each two-shell collision, we may estimate each spectrum by dividing the timeintegrated spectrum by the number of sub-pulses in the light curve. However, this method usually overestimates the target photon density because several sub-pulses may often overlap. In addition, observed pulse durations and intervals may not be ideal sub-pulse durations (that are directly calculated in the internal shock model) due to the overlap. In particular, high-energy gamma rays related to the maximum cutoff come from the outer collision with a wide sub-pulse, which 


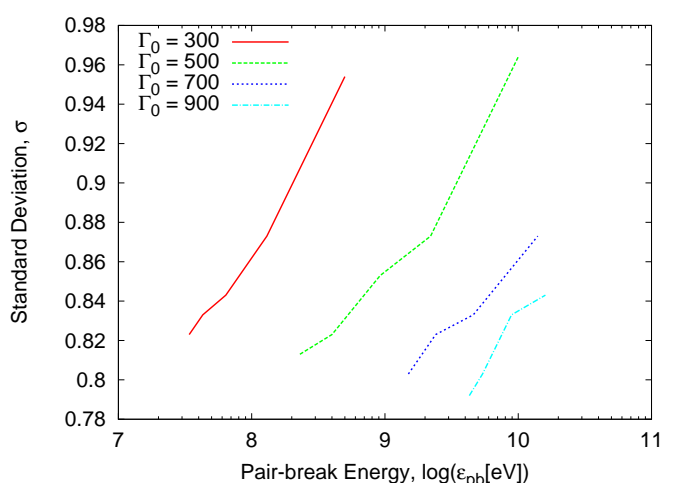

Fig. 6.- Pair-break energy vs. standard deviation of the sub-pulse interval distribution. The pair-break energy is defined by the energy where the spectrum becomes steeper than $\beta$ by 0.05 . We calculate these values by changing $\Gamma_{0}$ and $A$, which are the parameters of the initial Lorentz factor distribution. The solid line is written for $\Gamma_{0}=300$ changing $A$ from 1 to 0.2 . The dashed line is written for $\Gamma_{0}=500$ changing $A$ from 1 to 0.2 . The dotted line is written for $\Gamma_{0}=700$ changing $A$ from 1.3 to 0.4 . The dot-dashed line is written for $\Gamma_{0}=900$ changing $A$ from 1.5 to 0.6. Other parameters are the same as the calculation in Figure 3(b)

would be buried in many other spiky sub-pulses. Hence, we expect that the cutoff smearing effect can generally reduce the conventional estimate of the Lorentz factor.

One may see the pair-break energy in highenergy spectra. The pair-break energy is almost the same as the minimum cutoff energy in twoshell collisions. Hence, the pair-break energy would be useful instead of the original cutoff energy, if it is clearly observed. Although its observational identification may not be easy, let us consider the case that it is accomplished. Then, we can apply the conventional procedure described by various authors (e.g., Lithwick \& Sari 2001; Murase \& Ioka 2008). From Equations (10) and (44), we have (for $\beta>2$ )

$$
\begin{aligned}
\epsilon_{e} E_{\mathrm{int}} & \simeq V K(1+z) \frac{\varepsilon_{b}^{2}}{\beta-2}, \\
n^{\prime}\left(\tilde{\varepsilon}^{\prime}\right) & \simeq \frac{K}{\Gamma} \frac{(1+z) \varepsilon_{b}}{\beta-1}\left(\frac{\tilde{\varepsilon}}{\varepsilon_{b}}\right)^{1-\beta} .
\end{aligned}
$$

By substituting these equations into Equation (3),

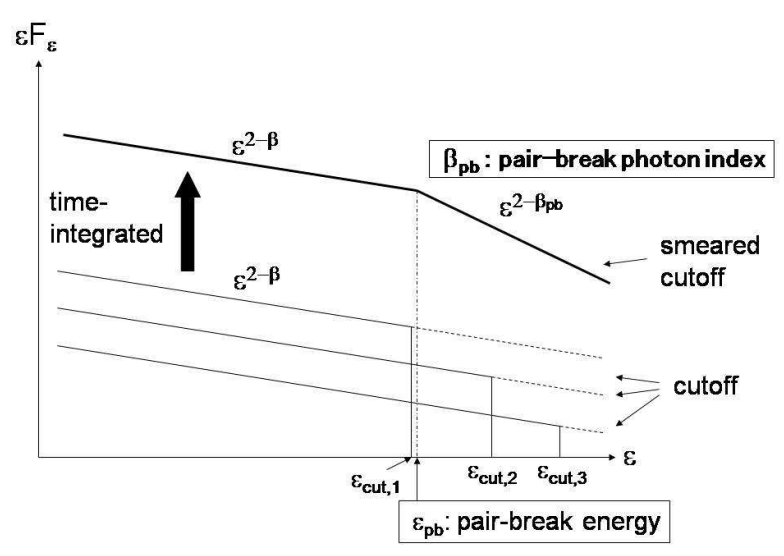

Fig. 7.- Schematic picture of the GRB spectrum. The cutoff is smeared by the time integration, although each energy spectrum for the two-shell collision has a cutoff. There is no cutoff in the timeintegrated spectrum but the slope becomes steep above the pair-break energy. This steep slope (the smeared cutoff) is made by the summation of different luminosity emissions. The pair-break energy is almost the same as the cutoff energy which originates from a two-shell collision at the most inner radius ( $\varepsilon_{\text {cut1 }}$ in this picture).

we obtain the Lorentz factor as

$$
\begin{aligned}
\Gamma \simeq & \left(\frac{\varepsilon_{\mathrm{pb}} \varepsilon_{\mathrm{b}}(1+z)^{2}}{\left(m_{e} c^{2}\right)^{2}}\right)^{\frac{\beta-1}{2 \beta+2}} \\
& \times\left(\frac{\xi \sigma_{T} \epsilon_{e} E_{\mathrm{int}}(1+z)^{2}(\beta-2)}{16 \pi c^{2} \varepsilon_{b} \delta t^{2}(\beta-1)}\right)^{\frac{1}{2 \beta+2}}
\end{aligned}
$$

where we use $V \simeq 4 \pi r^{2} \Delta$ and $r \sim 2 \Gamma^{2} \Delta \sim$ $2 \Gamma^{2} c \delta t /(1+z)$ expected in the internal shock model (and we can also expect $r \simeq 2 \Gamma^{2} c \tilde{\delta} t /(1+z)$ if an observed pulse duration $\tilde{\delta} t$ is the pulse decay time due to the curvature effect). For typical parameters, we have

$$
\begin{aligned}
\Gamma \simeq & 3.2 \times 10^{2}\left(\frac{\varepsilon_{\mathrm{pb}}}{500 \mathrm{MeV}}\right)^{\frac{3}{16}}(1+z)^{\frac{11}{16}}\left(\frac{\delta t}{0.1 \mathrm{~s}}\right)^{-\frac{5}{16}} \\
& \times\left(\frac{\epsilon_{e} E_{\mathrm{int}}}{10^{53} \mathrm{erg}}\right)^{\frac{5}{32}} \quad\left(\beta=2.2, \varepsilon_{b}=300 \mathrm{keV}\right),(8)
\end{aligned}
$$

where we note that $\epsilon_{e} E_{\text {int }}$ is the released radiation energy per collision.

How could we estimate the Lorentz factor from the time-integrated spectrum? First, we could 
roughly estimate the released energy from each collision, $\epsilon_{e} E_{\text {int }}$ by dividing the time-integrated spectrum by the sub-pulse number of the light curve, which may be observed in lower energy range near $\varepsilon_{b}$, once $D$ is determined by other observations. We could also estimate $\delta t /(1+z) \sim$ $l / c \sim d / c \sim \Delta t$ if we can know the sub-pulse width in light curves, although it is difficult to evaluate these values accurately from observations. Then, we could roughly estimate the Lorentz factor $\Gamma$ from the pair-break energy $\varepsilon_{\mathrm{pb}}$. One advantage of using the pair-break energy is that the minimum cutoff energy, i.e., the pair-break energy, is predominantly produced by the inner collision with a short sub-pulse width, which may be directly identified with reasonable values of the observed shortest variability timescale. Another advantage is that the pair-break energy is smaller than the cutoff energy in general and it can be smaller than the CIB attenuation energy. Several sub-pulses still often overlap, which may lead to our overestimating the released energy from each collision $\epsilon_{e} E_{\text {int }}$ via overestimating the duration and interval of sub-pulses from the most inner radii. Hence, as long as we can choose a reasonable shortest value of $\delta t$, this method could give an upper limit of the Lorentz factor (i.e., the true value of the Lorentz factor $\Gamma_{\text {true }}$, which is obtained considering masked sub-pulses, is related with the Lorentz factor calculated by our formulation $\Gamma_{\text {upper }}$ and a conventional estimate of the Lorentz factor $\Gamma_{\text {co }}$ as follows: $\Gamma_{\text {true }} \leq \Gamma_{\text {upper }} \leq \Gamma_{\text {co }}$ ). Again, note that conventional estimate generally lead to overestimating the minimum Lorentz factor when we use short timescales of $\delta t$ since true sub-pulses related to the high-energy emission may be wide and masked by other sub-pulses.

In the internal shock model, the Lorentz factor is likely to have a large dispersion, i.e., large $A$. Since the dispersion of the Lorentz factor $A$ is reflected by the dispersion of the sub-pulse interval $\sigma$, we may expect some correlation between $\sigma$ and $\varepsilon_{\mathrm{pb}}$ (see Figure 6). Although this $\sigma$ is not the observed dispersion itself which depends on algorithms determining pulse widths (e.g., Li \& Fenimore (1996)), it suggests that we can obtain some potential clue to the average value of the Lorentz factor from high-energy gamma-ray observations.

\subsection{Comparison with Analytical Calcula- tion}

We can calculate a power-law index produced by the smeared cutoff analytically if an initial variance of the sub-shells' Lorentz factor distribution $\sigma_{\Gamma, 0}^{2}$ satisfies $\sigma_{\Gamma, 0}^{2}<\Gamma_{0}^{2}$. Here $\Gamma_{0}$ is an average Lorentz factor and $\sigma_{\Gamma, 0}^{2}$ is defined in the source frame. Other assumptions are the same as the numerical calculation in this study. In this case, the variance of the shells' velocity distribution $\sigma_{v}$, which is defined in the center of mass, evolves with a radius of the expanding shell $r$ as $\sigma_{v} \propto r^{-1 / 3}$. The internal energy that originates from the two-shell collision is proportional to $\sigma_{v}^{2}$ (Beloborodov 2000) and this energy decreases as $E_{\text {int }} \propto \sigma_{v}^{2} \propto r^{-2 / 3}$ with radius (Li \& Waxman 2008). The internal energy is converted to nonthermal energy of electrons that radiate photons. We discuss a situation that a photon whose energy is $\varepsilon_{\text {cut }}$ annihilates with a photon whose energy is $\tilde{\varepsilon}\left(>\varepsilon_{b}\right)$. Here, $\tilde{\varepsilon}$ is the typical energy of target photons. Then the target photon number density is written as $n \propto E_{\mathrm{int}} \tilde{\varepsilon}^{1-\beta} / 4 \pi r^{2} \Delta \Gamma^{2} \propto$ $r^{-8 / 3} \tilde{\varepsilon}^{1-\beta} \Delta^{-1}$. We can calculate how the optical depth depends on $r$ and $\tilde{\varepsilon}, \tau_{\gamma \gamma}(\varepsilon) \propto n \sigma_{T} \Delta \propto$ $\tilde{\varepsilon}^{1-\beta} r^{-8 / 3}$. The target photon energy scales as $\tilde{\varepsilon} \propto r^{8 / 3(1-\beta)}$. High-energy photons with en$\operatorname{ergy} \varepsilon$ that satisfies $\varepsilon \geq\left(\Gamma m_{e} c^{2}\right)^{2} /(1+z) \tilde{\varepsilon}$ produce pairs in interaction with photons. We evaluate $\varepsilon_{\text {cut }}$ using the minimum energy for the paircreation threshold. Then, the cutoff energy scales as $\varepsilon_{\text {cut }} \propto r^{-8 / 3(1-\beta)}$. As we explain above, the internal energy which associates with the fluctuation of shell velocities decreases with shell's radius as $E_{\text {int }} \propto r^{-2 / 3}$. Hence $E_{\text {int }}$ relates with the cutoff energy as $E_{\text {int }} \propto \varepsilon_{\text {cut }}^{(1-\beta) / 4}$. The energy flux at $\varepsilon_{\text {cut }}$ is in proportion to $E_{\text {int }}$ and $\varepsilon_{\text {cut }}^{2-\beta}$ if we assume the energy spectrum that originates from two-shell collision satisfies the power law and the break energy (or peak energy) of each shell's spectrum does not change. The high-energy part of time-integrated spectrum would be

$$
\varepsilon F_{\varepsilon}(\varepsilon) \propto E_{\mathrm{int}} \varepsilon^{2-\beta} \propto \varepsilon^{\frac{9-5 \beta}{4}} .
$$

The pair-break photon index $\beta_{\mathrm{pb}}$ (see Figure 7) becomes $(5 \beta-1) / 4$ and the change of the index is $(\beta-1) / 4(=0.3$ for $\beta=2.2)$.

For large fluctuation of the Lorentz factor $\sigma_{\Gamma, 0}^{2}>\Gamma_{0}^{2}$, i.e., $A>1$, we cannot apply the above 
analytical results. Actually we have shown that the steepening is more mild for larger $A$ in Section 3

In Figure 3, we plot the time-integrated spectrum. The beginning energy of steepening depends on the fluctuation of the initial Lorentz factor $A$. The steepening begins at low energy when $A$ is large. The collision radius is small for large $A$ and the density of photons becomes large. This leads to the small cutoff energy of the two-shell collision and the steepening begins at lower energy. For large $A$, the steepening is small at the pair-break energy (see Section 3), but the slope becomes steep gradually at high energy because the fluctuation of Lorentz factor becomes small as shells collide with each other and the slope approaches the behavior of the analytical solution. In fact, $E_{\text {int }}$ follows the analytical solution, $\propto r^{-2 / 3}$, after some collisions. However, there is little difference between the numerical calculation and the analytical one even if the slope becomes steep. This can be understood as follows (see also Section (3). Photons whose energy is lower than $\varepsilon_{b}$, which is defined in Section 2.1. is important when the cutoff energy is large. The number of these photons are small to interact with other photons and the cutoff energy becomes large suddenly. In our numerical calculation, we approximate that there is no cutoff when the cutoff takes such a large value. On the other hand, we consider a single power law without the spectrum break at $\varepsilon_{b}$ in the analytical calculation. In this case, there are many low-energy photons and the cutoff energy increases following the power law of the collision radius. We checked that the numerical calculation gives the same result as the analytical calculation in a low $A$ regime when we assume the photon spectrum satisfies a single power law for a test numerical calculation. We can understand the behavior of the smeared cutoff in Figure 2 similarly. The pair-break photon index which is defined between $1 \mathrm{GeV}$ and $10 \mathrm{GeV}$ is 0.4 for $L_{\gamma}=10^{53} \mathrm{erg} \mathrm{s}^{-1}, 0.3$ for $L_{\gamma}=10^{52} \mathrm{erg} \mathrm{s}^{-1}$, and 0.3 for $L_{\gamma}=10^{51} \mathrm{erg}$ $\mathrm{s}^{-1}$, respectively.

\subsection{Comparison with Recent Observa- tions of Fermi}

We can estimate the Lorentz factor of a GRB if we observe a cutoff in a single sub-pulse spectrum. Then one may think that the maximum energy of the observed photon gives a lower limit of the Lorentz factor (Baring \& Harding 1997; Lithwick \& Sari 2001; Razzaque et al. 2004). However, as we have discussed in Section 4.1, the cutoff smearing effect generally allows lower Lorentz factor than the conventional estimates.

In the case of GRB080916C, $3 \mathrm{GeV}$ photon was detected by Fermi Large Area Telescope (LAT) in first $10 \mathrm{~s}$ and $13 \mathrm{GeV}$ photon was detected in $100 \mathrm{~s}$ (Abdo et al. 2009). In Abdo et al. (2009), there are five bins $(a-e)$ in the light curve. The spectrum index is almost constant from time interval $b$ to $e$. Hence, we can interpret the observation of beta as constant in one time interval of the observation, and it seems to be reasonable to interpret like this. We use the time interval $b$ to estimate the Lorentz factor in a following discussion. We can constrain the Lorentz factor using the maximum energy of a detected photon. The minimum Lorentz factor is 890 when we use following parameters, $\varepsilon_{b}=1.17 \times 10^{6} \mathrm{eV}, \varepsilon=3 \times 10^{9} \mathrm{eV}$, $\beta=2.21, z=4.35, E_{\text {int }}=4.2 \times 10^{54} \mathrm{erg}$ and $\delta t=2 \mathrm{~s}$. Here, the observed time interval $\tilde{\delta} t=2 \mathrm{~s}$ is regarded as the single sub-pulse width $\delta t$ with energy $E_{\text {int }}$. However, there may be a pair-break energy because the spectral steepening (by less than $(\beta-1) / 4 \sim 0.3)$ is too small to be detected with the current high energy data. When we set the sub-pulse duration to the high temporal variability, $\delta t \sim 100 \mathrm{~ms}$ detected by International Gamma-Ray Astrophysics Laboratory (INTEGRAL; Greiner et al. 2009) and $E_{\text {int }}=4 \times 10^{52}$ erg accordingly, the conventional estimate on the minimum Lorentz factor gives $\sim 1100$. However, this value may be overestimated. Here, let us apply our estimate using the pair-break energy. Then, we can obtain the Lorentz factor as $\sim 600\left(\varepsilon_{\mathrm{pb}} / 100 \mathrm{MeV}\right)^{3 / 16}$ for $E_{\mathrm{int}}=7 \times 10^{52} \mathrm{erg}$ and $\delta t=100 \mathrm{~ms}$. The actual Lorentz factor could be even lower as long as our choice of $\delta t$ is reasonable, because the spectrum consists of many sub-pulses in the cutoff smearing picture leading to overestimating the single sub-pulse energy $E_{\text {int }}$ (see Equations (7) and (8)).

A hard lag is observed in the observation of GRB080916C. It might suggest that the time development of a cutoff energy. Generally, the cutoff energy from an inner radius is smaller than that from an outer radius. The hard lag might be explained by multiple collision of shells because a 
photon from a inner radius seems to be detected first. However, we did not find the time lag in the different energy range in our calculation. A photon from an inner radius can be detected after a photon from an outer radius because the shell is distributed with a moderate width. It does not seem so easy to reproduce the time lag in the current model.

\subsection{Effects of Radiation and Acceleration Mechanisms}

In this study, we do not consider an additional high-energy component in the prompt emission spectrum. Such an additional component has been often expected in the optically thin synchrotron scenario (e.g., Mészáros 2006; Zhang 2007). Theoretically, there are two main classes as high-energy emission mechanisms, i.e., leptonic and hadronic mechanisms. The leptonic mechanisms include synchrotron self-Compton (SSC) emission and external inverse-Compton emission, which are the most discussed scenarios. Highenergy SSC emission is produced by relativistic electrons that radiate seed synchrotron photons themselves (e.g., Papathanassiou \& Mészáros 1996; Dai \& Lu 2002; Guetta \& Granot 2003). In addition, there may be some possibilities for external inverse-Compton emission via, e.g., upscattering thermal photons in the cocoon or nonthermal photons radiated from inner shells. In the later case, the integrated spectrum becomes softer than that of our model $(\mathrm{Li} 2010)$. The hadronic mechanisms include synchrotron radiation of high-energy baryons, synchrotron radiation of the secondary leptons generated in photohadronic interactions, as well as the photons directly produced from $\pi^{0}$ decays. In order to see the baryon synchrotron radiation, sufficiently strong magnetic fields are typically required (e.g., Gupta \& Zhang 2007; Murase et al. 2008). Otherwise, photohadronic components would dominate over the baryon synchrotron component as long as the photon density is high enough (Dermer \& Atovan 2004; Asano \& Inoue 2007). Hadronic gamma rays can be observed only when the non-thermal baryon loading is large enough (e.g. Murase \& Nagataki 2006; Asano \& Inoue 2007). The above mechanisms can lead to an additional high-energy component that can be observed by Fermi, and high-energy spectra may differ from those demonstrated in this work. Although they are potentially important, we postpone detailed discussions in this work for our demonstrative purpose.

Another uncertainty raises from the origin of the cutoff in high-energy spectra. For example, the maximum energy of generated photons is at most the maximum energy of electrons, which is often determined by comparing between the synchrotron cooling time and acceleration time in the optically thin synchrotron scenario. When the SSC emission is not significant, if the paircreation opacity is small enough or the acceleration efficiency is small enough, the cutoff would be determined by the electron maximum energy rather than the pair-creation process (e.g., Ioka 2010). In addition, other processes such as the Thomson scattering may also be relevant (e.g., Pe'er \& Waxman 2004). Also, the acceleration mechanism affects the electron spectrum itself as well as the maximum energy of electrons. Generally speaking, the relativistic diffusive shock acceleration sensitively depends on properties of magnetic fields around shocks, so that the spectral index may not be universal and the spectrum may differ from the simple power law (e.g., Achterberg et al. 2001; Blasi \& Vietri 2005; Lemoine \& Revenu 2006; Niemiec \& Ostrowski 2006; Morlino et al. 2007a b; Aoi et al. 2008). These effects from radiation and acceleration mechanisms may significantly affect our results of high-energy spectra made by the smeared paircreation cutoff.

\subsection{Effects of radiative transfer}

In this study, we calculated the energy spectrum assuming that each shell is regarded as a time-independent one-zone shell. One may think about effects of spatially distributed photons and/or time-dependent formulations. However, our conclusion does not change so much even if we consider these effects. We describe explanations below. First, let us consider the effect of the spatially distributed photons. High-energy photons are preferentially weighted by contributions of outer regions because photons of outer regions typically have the lower optical depth for paircreation processes. One obtains a broken powerlaw energy spectrum even for emission from a single shell, which is different from the spectrum with 


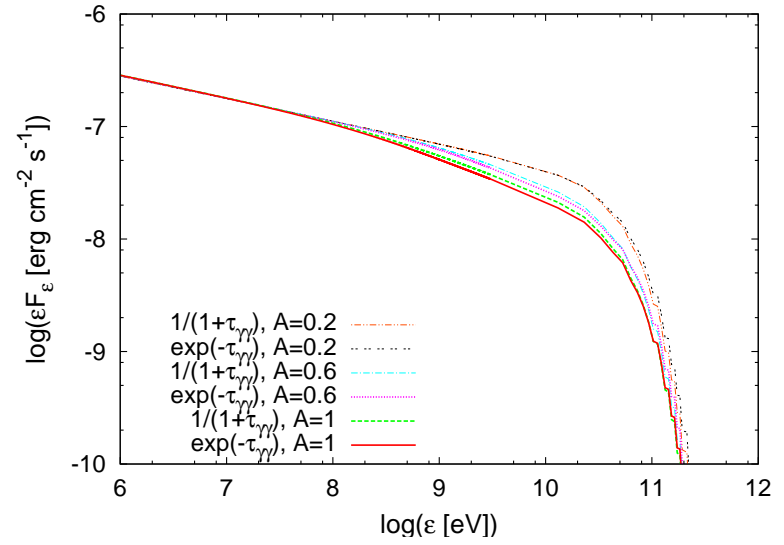

Fig. 8. - Time-integrated spectra for two models. Curves labeled as $\exp \left(-\tau_{\gamma \gamma}\right)$ represent the spectrum that is summed over the spectra with the exponential cutoff. Curves labeled as $1 /\left(1+\tau_{\gamma \gamma}\right)$ represent the spectrum that is summed over the spectra with the $1 /\left(1+\tau_{\gamma \gamma}\right)$ attenuation signature. Curves show similar profile. Parameters are the same as the calculation in Figure 3(b).

the exponential cutoff (see Section 2.2). However, we can argue that the result in the overall spectrum does not change dramatically even if we use the exponential cutoff. Let us examine the case of $1 /\left(1+\tau_{\gamma \gamma}\right)$ attenuation signature, which is valid when we consider the effect of spatially distributed photons (e.g., Baring 2006). $\tau_{\gamma \gamma}$ is in proportion to $\varepsilon^{\beta-1}$ in our model (see Equations 3 and [6). Thus, a slope of a single-shell energy spectrum changes from $-\beta$ to $-2 \beta+1$ at $\varepsilon_{\text {cut }}$. As a result of our calculation, the slope of the time-integrated spectrum changes from $-\beta$ to $-(5 \beta-1) / 4$ by the effect of multiple shells even if fluctuation of the initial Lorentz factor distribution $A$ is small (see Section 4.2). The change of the slope becomes smaller if $A$ is larger. Hence the consequence of $1 /\left(1+\tau_{\gamma \gamma}\right)$ attenuation signature is masked by the effect of multiple shells as far as $\beta>1$, because the change of slope due to $1 /\left(1+\tau_{\gamma \gamma}\right)$ attenuation is larger than that due to the effect of multiple shells. This means that the exponential cutoff and $1 /\left(1+\tau_{\gamma \gamma}\right)$ attenuation gives similar results, and our conclusion does not depend on the choice. In fact, the exponential cutoff and $1 /\left(1+\tau_{\gamma \gamma}\right)$ give similar spectra in Figure 8 Note that the exponential turnovers in this figure come from the attenuation by the CIB. Two spectra are almost same for small $A$. On the other hand, there is small difference in a high-energy range when the above analytical solution is not good (i.e., large $A)$. The spectrum with $1 /\left(1+\tau_{\gamma \gamma}\right)$ attenuation signature is larger than that with the exponential cutoff. This is because emission from inner radius is large for large $A$ and a $1 /\left(1+\tau_{\gamma \gamma}\right)$ tail is comparable to a spectrum of emission from outer radius. Another effect of spatially distributed photons also arises if electrons are in the fast cooling range and particles are accelerated by the diffusive shock acceleration, since such electrons are expected to be confined to the emission region close to the shock. The localization of the fast cooling electrons may affect our results (Granot et al. 2008).

Next, we examine the effect of a time-dependent formulation. There is an intermediate power-law segment in the time integrated spectrum when we consider the time development of the photon field and the geometrical effect, under the assumption that a thin shell expands and emits isotropically in its own rest frame (Granot et al. 2008). The slope of the spectrum is $-\beta, 1-2 \beta$ and $-(\beta-1)(2 \beta+3) /(\beta+1)-1$, respectively, for impulsive sources (i.e., for thin shells). The slope of the intermediate power law is steeper than that of the spectrum integrated over multiple shells. Then, the time-integrated spectrum resulting from our calculation would not be changed as well as we discussed in the last paragraph for the case of the spatially distributed photons, if their formulation is used. This is because a change of the slope by the time-dependent effect is steeper than that by the effect of multiple shells. Note that the first break energy (labeled as $\varepsilon_{1 i}$ in Granot et al. 2008) with the time-dependent calculation is equal to $\varepsilon_{\text {cut }}$ determined from time-independent one-zone calculation. The difference between the two formulations is masked once we integrate spectra of photons emitted from each shell, so that our conclusion does not change so much by this effect as long as we suppose impulsive sources.

Note that the above discussions are correct under our assumption (see Section 21). The effects of the radiative transfer may not be remarkable unless photons are not emitted from the outer region effectively (i.e., the effect of the spatially distributed photons and/or the time-dependent are weaker than that of the multiple shells). For example, an amount of high-energy photons becomes 


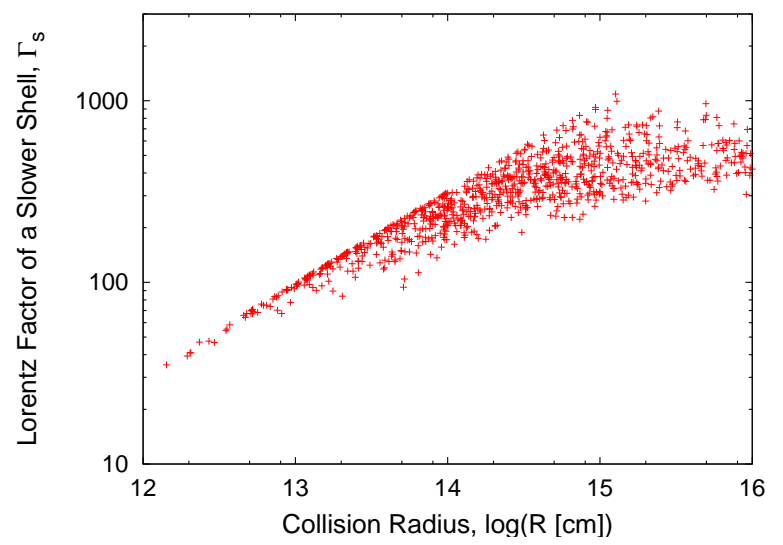

Fig. 9.- Collision radius vs. a Lorentz factor of a slower shell. These two values are correlated with each other when an initial fluctuation of Lorentz factor $A$ is large. We calculated these values for $A=1$. Other parameters are the same as the calculation in Figure 3(a).

less if $\beta$ and $\varepsilon_{b}$ decrease as a collision radius becomes large.

Finally, let us discuss the effect of the opacity by outer shells. Photons emitted from inner shells are absorbed in outer shells if $\varepsilon_{\text {cut }}$ of photons emitted from inner shells are larger than that from outer shells. However, $\varepsilon_{\text {cut }}$ is small at inner shells if shells' Lorentz factors are small in an inner radius. This trend is obvious for large $A$ because a Lorentz factor distribution of shells becomes more important than a spatial distribution of shells to determine a collision radius. In other words, a shell whose Lorentz factor is sufficiently small collides with another shell in an inner region even if an initial position of this shell is relatively outer region compared with other shells. Note that the collision radius is determined by a velocity of a caught-up shell (i.e., a slower shell) and the distribution of collision radii is correlated with the distribution of slower shells (Figure 9). On the other hand, a correlation between the Lorentz factor and a collision radius is weak for small $A$ because the effect of the spatial distribution is remarkable. Hence, when $A$ is large, outer sub-shells do not contribute to the pair-creation opacity for photons emitted from inner sub-shells unless energy of radiated photons from outer radii is rather larger than that from inner radii. Otherwise, photons from inner subshells suffer from $e^{-\tau_{\gamma \gamma}}$ in outer sub-shells.

\section{Summary and Conclusion}

We have investigated the spectral features of the electron-positron pair creation in the GRB prompt emission to probe the Lorentz factor of the GRB outflow. We have studied the timeintegrated spectrum from many internal shocks with numerical and analytical calculations. This is because almost all the authors studied the paircreation cutoff energy expected from one emission region, although it could be difficult to observe the emission from a single collision. Although the cutoff energy may be observed for a simple subpulse from bright bursts and it is very useful (e.g., Murase \& Ioka 2008; Gupta \& Zhang 2008), it is also important to study whether the cutoff can be observed or not for bursts that have many subpulses in their light curves. Fermi typically allows us to obtain the high-energy spectrum integrated over some time interval (e.g., $\tilde{\delta}=2 \mathrm{~s}$ ) in many cases due to the difficulty to collect many highenergy photons and the overlapping of sub-pulses. In this sense, current and future Cherenkov detectors with the low-energy threshold of a few $\times 10 \mathrm{GeV}$ are important to study the cutoff because these observatories can collect many highenergy photons. MAGIC and VERITAS should be useful for nearby GRBs, and future CTA will allow us to have more chances to observe GRBs with high-energy emission.

We demonstrate that the clear cutoff, which comes from electron-positron pair-creation processes, is hard to observe in the internal shock model, when bursts consist of many sub-shell collisions. Instead, the slope of the spectrum becomes steeper above the energy, so-called the pairbreak energy $\varepsilon_{\mathrm{pb}}$, which also originates from the electron-positron pair creation. We have demonstrated how the cutoff is smeared, and compared our numerical results with analytical consideration. Possibly, we expect that Fermi observes this pair-break energy in the high-energy range. The pair-break energy may be observable if the luminosity is greater than $\sim 10^{52} \mathrm{erg} \mathrm{s}^{-1}$ and the fluctuation of the initial Lorentz factor $A$ is large for $z=1$. However, the change of the spectrum slope is small in general and it may not be easy to observe the pair-break energy. Hence, observing nearby GRBs is important to identify the pair-break energy. It is also important to identify 
the pair-break energy by multi-wavelength observation using Cherenkov detectors. If it is detected, we have some information on the Lorentz factor of the shell emitting photons with $\sim \varepsilon_{\mathrm{pb}}$.

It was conventionally discussed that the paircreation cutoff is a broken power law (not an exponential one) since the observed gamma rays and target photons are co-spatially and/or timedependently distributed in a single collision. However, our multiple shell effect would be more prominent and mask the above effect with smaller change of the power-law index as long as $\beta>1$ and we suppose impulsive sources.

The cutoff smearing effect can generally reduce the conventional estimate of the Lorentz factor. We discuss the possibility that the maximum energy of detected photons does not provide the best indicator of the minimum Lorentz factor. The pair-break energy is also easily missed because the steepening of the spectral index is relatively small (less than $(\beta-1) / 4 \sim 0.3$ ). Then the current observations still allow smaller Lorentz factor than the previous estimates. We have applied the cutoff smearing effect to GRB080916C and suggested that the observations are consistent with the Lorentz factor of $\sim 600$ (and even smaller value) that is slightly below the previous result of $\sim 900$.

The Lorentz factor of the outflow is likely to have a large dispersion in the internal shock model. When $A$ is large, the resulting spectrum has lower pair-break energy where the smearing starts, and vice versa. This suggests that dispersion of observed sub-pulses may have some clues to estimate the Lorentz factor of the outflow.

There are differences between the results of the numerical calculation and those of an analytical one, although the analytical calculation is a good approximation for small fluctuation of an initial Lorentz factor. The steepening is small in the low energies for large $A$, compared with the analytical calculation. However, numerical values approach analytical values in the high energies by the effect of multiple collisions.

Although we have demonstrated that the smeared cutoff behavior is characterized by steepened power-law spectra, there are a lot of uncertainties in the predicted high-energy spectra even in the internal shock model (see Sections 4.4 and
4.5). (1) We have fixed $\varepsilon_{b}, \epsilon_{e}$ and $\beta$, but changing either of them for each collision easily changes the resulting spectra. Even the power-law approximation for accelerated electrons may not be good, because it depends on the unknown acceleration mechanism. (2) There may be an additional highenergy component coming from e.g., the inverseCompton scattering and/or baryon-initiated electromagnetic cascade processes. (3) The cutoff may originate from other processes such as the maximum energy of accelerated electrons. There is also some uncertainty in the high energies where attenuation by the CIB is important due to the ambiguity of the CIB. Although the smeared cutoff spectrum may easily change if we consider the above effects, it is commonly expected that the obvious cutoff cannot be seen for time-integrated spectra of bursts with many sub-pulses in light curves, as long as we believe the internal shock model. Such a smearing effect is also expected for other high-energy features. For example, a part of the authors provided the recipe to diagnose the mechanism of prompt emission for individual subpulses and discussed the pair annihilation bump (Murase \& Ioka 2008). We can expect the smearing effect if photospheric emission is also caused by the internal shock dissipation. Even high-energy neutrino spectra (see for high-energy neutrinos, e.g., Murase (2007), and references there in) can be modified since the magnetic field will be weaker at larger emission radii that can affect some of the previous results (Asano \& Nagataki 2006). The recently launched Fermi can be useful for testing the smeared cutoff spectrum. However, it seems that we still need many $>\mathrm{GeV}$ photons for further investigations. Therefore, not only Fermi but also current Cherenkov detectors such as MAGIC or planned low-energy threshold Cherenkov detectors such as CTA and 5@5 will be important. Once we see high-energy events by these larger area telescopes, good photon statistics will allow us to study the high-energy behavior of prompt emission well.

Recently, Zhang \& Pe'er argued that the emission radius of GRB080916C should be larger than $r \gtrsim 10^{15} \mathrm{~cm}$. Our estimate based on the internal shock model suggests $r \gtrsim 10^{14.5} \mathrm{~cm}$. Since the true Lorentz factor in the multi-zone scenario can be smaller than in the one-zone scenario, even smaller emission radii are possible. They also argued that 
the pure fireball model may not avoid the photospheric emission as the relic thermal emission. Note that, even if the outflow is initially Poyntingdominated, the internal shock model may still be one of the viable models as long as a significant fraction of the magnetic energy can be converted into kinetic energy (Vlahakis \& Köumlnigl 2003). Recently, models that GRB emission is powered by dissipation of the Poynting flux energy within the outflow have been discussed more and more (Lvutikov \& Blandford 2003; Naravan \& Kumar 2009). Future observations of broadband spectra that are well time resolved will give us very crucial clues to the realistic GRB prompt emission model.

The authors thank the anonymous referee for useful suggestions that improved this paper. J.A. thanks Y. Sendouda for fruitful discussions. This research was supported by a Grant-in-Aid for the Global COE Program The Next Generation of Physics, Spun from Universality and Emergence from Ministry of Education, Culture, Sports, Science and Technology (MEXT). J.A. and K.M. are supported by Grants-in-aAid for Japan Society for the Promotion of Science (JSPS) Fellows from MEXT. K.T. is supported in part by Monbukagaku-sho Grant-in-Aid for the global COE programs, Quest for Fundamental Principles in the Universe: from Particles to the Solar System and the Cosmos, at Nagoya University. This research was supported by Grant-in-Aid for Scientific Research on Priority Areas from MEXT 19047004 (K.I. and S.N.), Grant-in-Aid for Scientific Research (S) 19104006 (S.N.) from JSPS, Grant-in-Aid for young Scientist(A) 21684014 (K.I.), and Grant-in-Aid for young Scientists(B) 18740147 (K.I.) and 19740139 (S.N.) from JSPS. The numerical calculations were carried out on Compaq Alpha Server ES40 at Yukawa Institute for Theoretical Physics, Kyoto University. 


\section{A. Two-Shell Interaction}

We consider the situation that a rapid shell collides with a slower shell. We assume two shells merge after collision. This assumption is valid when the merged shell becomes cool immediately (i.e., the cooling time by emission is shorter than the dynamical time in which a shock wave crosses a shell). We calculate the physical quantities of the merged shell defined in the source frame, Lorentz factor $\Gamma_{m}$, the internal energy $E_{\text {int }}$, number density of protons $n_{p, m}$, thickness of the shell $l_{m}$, and area $\Sigma_{m} \simeq 4 \pi r^{2}$ using initial values of Lorentz factor $\Gamma$, density of protons $n_{p}$, thickness $l$, and area $\Sigma \simeq 4 \pi r^{2}$ (i.e., volume of the shell is $V \simeq \Sigma l$ ) of two shells. Physical quantities of a rapid (slower) shell is denoted by the subscript "r" (" $s$ "). The kinetic energy of two shells converts to the internal energy when two shells collide and shock waves occur. Using conservation of momentum and energy, we can calculate the velocity and the internal energy of the merged shell:

$$
\begin{gathered}
\Gamma_{m} \simeq \sqrt{\frac{m_{r} \Gamma_{r}+m_{s} \Gamma_{s}}{m_{r} / \Gamma_{r}+m_{s} / \Gamma_{s}}}, \\
E_{\text {int }}=m_{r} c^{2}\left(\Gamma_{r}-\Gamma_{m}\right)+m_{s} c^{2}\left(\Gamma_{s}-\Gamma_{m}\right),
\end{gathered}
$$

where $m=m_{p} n_{p} V$ ( $m_{p}$ is the proton mass). This internal energy is radiated immediately. We calculate the energy spectrum of the emission in Section 2.1.

Two shock waves occur when shells merge, a forward shock and a reverse shock. The velocity of a forward (reverse) shock $\Gamma_{\mathrm{fs}}\left(\Gamma_{\mathrm{rs}}\right)$ is written as $\Gamma_{\mathrm{fs}} \simeq \Gamma_{m} \sqrt{\left(1+2 \Gamma_{m} / \Gamma_{s}\right) /\left(2+\Gamma_{m} / \Gamma_{s}\right)}\left(\Gamma_{\mathrm{rs}} \simeq \Gamma_{m} \sqrt{\left(1+2 \Gamma_{m} / \Gamma_{r}\right) /\left(2+\Gamma_{m} / \Gamma_{r}\right)}\right)$ (Sari \& Piran 1995; Kobavashi et al. 1997). These shocks compress the initial shells and the thickness of the merged shell $l_{m}$ is given by

$$
l_{m}=l_{s} \frac{\beta_{\mathrm{fs}}-\beta_{m}}{\beta_{\mathrm{fs}}-\beta_{s}}+l_{r} \frac{\beta_{m}-\beta_{\mathrm{rs}}}{\beta_{r}-\beta_{\mathrm{rs}}} .
$$

For simplicity, we assume the density of the merged shell becomes homogeneous, although there is the contact discontinuity in fact. We use the averaged density written as:

$$
n_{p, m}=\frac{n_{p, r} l_{r}+n_{p, s} l_{s}}{l_{m}}
$$

\section{B. Emission from Multiple Shells}

We consider the multiple shells colliding with each other and the emission from the merged shell. We can calculate the energy spectrum of the emission by applying the discussion of the previous section to each collision.

We calculate the dynamics of shells in one dimension numerically (Kobavashi et al. 1997). We consider $N$ shells which are labeled by an index $i(i=1, \ldots, N)$, where the inner shell is labeled by the larger number. These shells are characterized by four variables, Lorentz factor $\Gamma_{i}$, density $n_{i}$, thickness of a shell $l_{i}$, and the initial position of (inner part of ) the shell $r_{i}$. We determine the initial position so as to the length between the two shells $d_{i}\left(=r_{i}-r_{i+1}-l_{i+1}\right)$ becomes equal (i.e., $d_{i}$ is same for all shells). We also assume $d_{i}$ is comparable to $l_{i}$ and set $d_{i}=5 \times l_{i}$. Lorentz factors are highly relativistic value and distributed randomly following the log-normal distribution. The log-normal distribution is defined by $\Gamma_{0}$ and the amplitude of the fluctuation $A$ as follows:

$$
\ln \frac{\Gamma-1}{\Gamma_{0}-1}=A x, \quad P(x) d x=\frac{e^{-x^{2} / 2}}{\sqrt{2 \pi}} d x .
$$

Its mean value is $\exp \left(\ln \left(\Gamma_{0}-1\right)+A^{2} / 2\right)$ and its variance is $\left(\exp \left(A^{2}\right)-1\right) \exp \left(2 \ln \left(\Gamma_{0}-1\right)+A^{2}\right)\left(\equiv \sigma_{\Gamma}^{2}\right)$. At $A<1$, we have $\left(\Gamma-\Gamma_{0}\right) / \Gamma_{0} \simeq A x$, then $\Gamma_{0}$ becomes the mean value and $\sigma_{\Gamma} / \Gamma_{0} \simeq A$. At $A>1$, we are in the high amplitude regime. The specific values of parameters are described in the caption of the figures. Note 
that we chose the width of the shell in order to set the variance time at about 10 or $100 \mathrm{~ms}$. At $t=0$ the shells are at the initial positions. These shells expand spherically with highly relativistic speed and density decreases in proportion to $r^{-2}$, where $r$ is the radius of the expanding shell. Strictly, radial velocity spread causes a gradual spread of radial width of shells at large radius. Although the shell spreading would be important at large radii, we do not consider the shell spreading in this paper for the ease of understanding. A rapid shell catches up with a slower one and both collide with each other. We assume two shells merge after collision and the shell becomes cold immediately via emission. We can calculate the internal energy which occurs in the $j$ th collision $E_{\text {int,j }}$ and the energy spectrum of photons $d n_{j} / d \varepsilon$ (see Section 2.1). We also calculate the position $R_{j}$ and the time $t_{j}$. An observer at $D$ away from the central source will begin to detect the emission at a time $t_{j, \mathrm{ob}}=\left[D-R_{j}\right] / c+t_{j}$.

Shells expand and collide with other shell one after another. This process continues until there is no rapid shell behind the slower shell or all shells merge into one shell. Note that we do not consider the interstellar medium (ISM). Most shells collide at the small radius where the effect of the ISM is negligible when we consider the large value of $A$. The shell collides at the large radius and the effect of the ISM is important and the shell would be decelerated when we consider low $A$.

We have the time-integrated energy spectrum $d n_{\text {sum }} / d \varepsilon=\Sigma_{j=1}^{N_{\text {coll }}} d n_{j} / d \varepsilon$, where $N_{\text {coll }}$ is the number of collisions. We focus on the time-integrated spectrum in this study. We define the duration $T$ of the GRB by the time we detect $95 \%$ of the total energy. We define the time-averaged luminosity $L_{\gamma}$ by $L_{\gamma}=$ $(1+z)^{2}\left[\int d \varepsilon \varepsilon\left(d n_{\text {sum }} / d \varepsilon\right)\right] / T$. 


\section{REFERENCES}

Abdo et al. 2009, Science, 323, 1688

Achterberg, A., Gallant, Y. A., Kirk, J. G., \& Guthmann, A. W. 2001, MNRAS, 328, 393

Aharonian, F., et al. 2006, Nature, 440, 1018

Aharonian, F., et al. 2007, A\&A, 475, L9

Albert, J., et al. 2007, ApJ, 667, 358

Aoi, J., Murase, K., \& Nagataki, S. 2008, MNRAS, 383, 1431

Asano, K., \& Inoue, S. 2007, ApJ, 671, 645

Asano, K., \& Nagataki, S. 2006, ApJ, 640, L9

Band, D., et al. 1993, ApJ, 413, 281

Baring, M. G. 2006, ApJ, 650, 1004

Baring, M. G., \& Harding, A. K. 1997, ApJ, 491, 663

Beloborodov, A. M. 2000, ApJ, 539, L25

Blasi, P., \& Vietri, M. 2005, ApJ, 626, 877

Bošnjak, Ž., Daigne, F., \& Dubus, G. 2009, A\&A, 498, 677

Dai, Z. G., \& Lu, T. 2002, ApJ, 580, 1013

Daigne, F., \& Mochkovitch, R. 1998, MNRAS, 296, 275

Daigne, F., \& Mochkovitch, R. 2000, A\&A, 358, 1157

Dermer, C. D., \& Atoyan, A. 2004, A\&A, 418, L5

Fan, Y.-Z., \& Piran, T. 2008, Frontiers of Physics in China, 3, 306

González, M. M., Dingus, B. L., Kaneko, Y., Preece, R. D., Dermer, C. D., \& Briggs, M. S. 2003, Nature, 424, 749

Gould, R. J., \& Schréder, G. P. 1967, Physical Review , 155, 1404

Granot, J., Cohen-Tanugi, J., \& do Couto e Silva, E. 2008, ApJ, 677, 92

Greiner, J., et al. 2009, A\&A, 498, 89
Guetta, D., \& Granot, J. 2003, ApJ, 585, 885

Gupta, N., \& Zhang, B. 2007, MNRAS, 380, 78

Gupta, N., \& Zhang, B. 2008, MNRAS, 384, L11

Hurley, K., et al. 1994, Nature, 372, 652

Ioka, K. 2010, arXiv:1006.3073

Ioka, K., Murase, K., Toma, K., Nagataki, S., \& Nakamura, T. 2007, ApJ, 670, L77

Ioka, K., \& Nakamura, T. 2002, ApJ, 570, L21

Kneiske, T. M., Bretz, T., Mannheim, K., \& Hartmann, D. H. 2004, A\&A, 413, 807

Kobayashi, S., Piran, T., \& Sari, R. 1997, ApJ, 490, 92

Kobayashi, S., \& Sari, R. 2001, ApJ, 551, 934

Lemoine, M., \& Revenu, B. 2006, MNRAS, 366, 635

Li, Z. 2010, ApJ, 709, 525

Li, H., \& Fenimore, E. E. 1996, ApJ, 469, L115

Li, Z., \& Waxman, E. 2008, ApJ, 674, L65

Liang, E. W., Dai, Z. G., \& Wu, X. F. 2004, ApJ, 606, L29

Lightman, A. P., \& Zdziarski, A. A. 1987, ApJ, 319,643

Lithwick, Y., \& Sari, R. 2001, ApJ, 555, 540

Lyutikov, M., \& Blandford, R. 2003, arXiv:astro-ph/0312347

McBreen, B., Hurley, K. J., Long, R., \& Metcalfe, L. 1994, MNRAS, 271, 662

Meszaros, P. 2006, Reports on Progress in Physics, 69,2259

Mimica, P., Aloy, M. A., Mueller, E. 2007, A\&A, 466,93

Morlino, G., Blasi, P., \& Vietri, M. 2007, ApJ, 658,1069

Morlino, G., Blasi, P., \& Vietri, M. 2007, ApJ, 662,980

Murase, K., \& Nagataki, S. 2006, PRL, 97, 051101 
Murase, K. 2007, Phys. Rev. D, 76, 123001

Murase, K., \& Ioka, K. 2008, ApJ, 676, 1123

Murase, K., Asano, K., \& Nagataki, S. 2007, ApJ, 671,1886

Murase, K., Ioka, K., Nagataki, S., \& Nakamura, T. 2008, Phys. Rev. D, 78, 023005

Murase, K., Zhang, B., Takahashi, K., \& Nagataki, S. 2009, MNRAS, 396, 1825

Nakar, E., \& Piran, T. 2002, MNRAS, 331, 40

Narayan, R., \& Kumar, P. 2009, MNRAS, 394, L117

Niemiec, J., \& Ostrowski, M. 2006, ApJ, 641, 984

Papathanassiou, H., \& Mészáros, P. 1996, ApJ, 471, L91

Pe'er, A., Mészáros, P., \& Rees, M. J. 2006, ApJ, 642, 995

Pe'er, A., \& Waxman, E. 2004, ApJ, 613, 448

Preece, R. D., Briggs, M. S., Mallozzi, R. S., Pendleton, G. N., Paciesas, W. S., \& Band, D. L. 2000, ApJS, 126, 19

Razzaque, S., Mészáros, P., \& Zhang, B. 2004, ApJ, 613, 1072

Rees, M. J., \& Mészáros, P. 2005, ApJ, 628, 847

Sari, R., \& Piran, T. 1995, ApJ, 455, L143

Schneid, E. J., et al. 1992, A\&A, 255, L13

Schneid, E. J., et al. 1995, ApJ, 453, 95

Sommer, M., et al. 1994, ApJ, 422, L63

Svensson, R. 1987, MNRAS, 227, 403

Takahashi, K., Murase, K., Ichiki, K., Inoue, S., \& Nagataki, S. 2008, ApJ, 687, L5

Thompson, C. 1994, MNRAS, 270, 480

Vlahakis, N. \& Köumlnigl, A. 2003, ApJ, 596, 1104

Wagner, R. M., Lindfors, E. J., Sillanpää, A., \& Wagner, S. 2009, arXiv:0912.3742
Wang, X.-Y., Li, Z., Dai, Z.-G., \& Mészáros, P. 2009, ApJ, 698, L98

Yonetoku, D., Murakami, T., Nakamura, T., Yamazaki, R., Inoue, A. K., \& Ioka, K. 2004, ApJ, 609, 935

Zhang, B. 2007, Chinese Journal of Astronomy and Astrophysics, 7, 1

Zhang, B., \& Pe'er, A. 2009, ApJ, 700, L65

Zou, Y.-C., Fan, Y.-Z., \& Piran, T. 2009, MNRAS, 396,1163

This 2-column preprint was prepared with the AAS LATEX macros v5.2. 